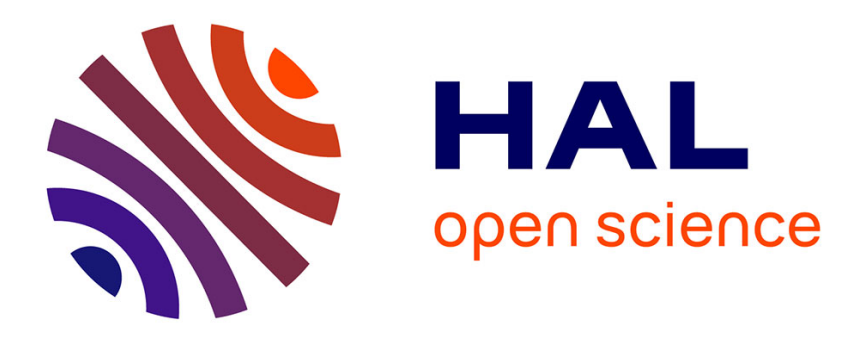

\title{
Quasi-Monte Carlo methods for Markov chains with continuous multi-dimensional state space
}

\author{
Rami El Haddad, Christian Lécot, Pierre L'Ecuyer, Nabil Nassif
}

\section{To cite this version:}

Rami El Haddad, Christian Lécot, Pierre L'Ecuyer, Nabil Nassif. Quasi-Monte Carlo methods for Markov chains with continuous multi-dimensional state space. Mathematics and Computers in Simulation, 2010, 81 (3), pp.560-567. hal-00949602

\section{HAL Id: hal-00949602 \\ https://hal.univ-grenoble-alpes.fr/hal-00949602}

Submitted on 19 Feb 2014

HAL is a multi-disciplinary open access archive for the deposit and dissemination of scientific research documents, whether they are published or not. The documents may come from teaching and research institutions in France or abroad, or from public or private research centers.
L'archive ouverte pluridisciplinaire HAL, est destinée au dépôt et à la diffusion de documents scientifiques de niveau recherche, publiés ou non, émanant des établissements d'enseignement et de recherche français ou étrangers, des laboratoires publics ou privés. 


\title{
Quasi-Monte Carlo methods for Markov chains with continuous multi-dimensional state space
}

\author{
R. El Haddad ${ }^{\mathrm{a}, *}$, C. Lécot ${ }^{\mathrm{b}}$, P. L’Ecuyer ${ }^{\mathrm{c}}$, N. Nassif ${ }^{\mathrm{d}}$ \\ ${ }^{a}$ Département de Mathématiques, Université Saint-Joseph, BP 11-514, Riad El Solh \\ Beyrouth 1107 2050, Liban \\ ${ }^{b}$ LAMA, UMR 5127 CNRS \&3 Université de Savoie, 73376 Le Bourget du Lac, France \\ ${ }^{c}$ Département d'Informatique et de Recherche Opérationnelle, Université de Montréal, \\ CP 6128, Succ. Centre-Ville, Montréal, H3C 3J7, Canada \\ ${ }^{d}$ Department of Mathematics, American University of Beirut, BP 11-0236, Riad El-Solh \\ Beyrouth 1107 2020, Liban
}

\begin{abstract}
We describe a quasi-Monte Carlo method for the simulation of discrete time Markov chains with continuous multi-dimensional state space. The method simulates copies of the chain in parallel. At each step the copies are reordered according to their successive coordinates. We prove the convergence of the method when the number of copies increases. We illustrate the method with numerical examples where the simulation accuracy is improved by large factors compared with Monte Carlo simulation.
\end{abstract}

Keywords: Markov chain, discrepancy, quasi-Monte Carlo method, simulation.

\section{Introduction}

Many real-life systems can be modeled using Markov chains. Fields of application are queueing theory, telecommunications, option pricing, etc. In most interesting situations, analytic formulas are not available and the state space of the chain is so large that classical numerical methods would require

\footnotetext{
*Corresponding author. Tel.: +961 (1) 421 372; fax: +961 (4) 532657

Email addresses: rami.haddad@fs.usj.edu.lb (R. El Haddad), christian.lecot@univ-savoie.fr (C. Lécot), lecuyer@iro.umontreal.ca (P. L'Ecuyer), nn12@aub.edu.lb (N. Nassif)
} 
a considerable computational time and huge memory capacity. So Monte Carlo (MC) simulation becomes the standard way of estimating performance measures for these systems. A drawback of MC methods is their slow convergence. One approach to improve the accuracy of the method is to change the random numbers used. Quasi-Monte Carlo (QMC) methods use quasirandom numbers instead of pseudo-random numbers. Pseudo-random numbers aim to simulate a sequence of independent and identically distributed (i.i.d.) random variables with a given distribution (we only consider the uniform distribution). In the example of $\mathrm{MC}$ integration, it is not so much the randomness of the samples that is relevant, but rather that the samples should be spread in a uniform manner over the integration domain. Quasirandom numbers are sample points for which the empirical distribution is close to the uniform distribution; unlike for random sampling, quasi-random points are not required to be independent and may be completely deterministic.

The efficiency of a QMC method depends on the quality of the quasirandom points that are used. Broadly speaking, these points should form a low-discrepancy point set. We recall from [12] some basic notations and concepts. We first denote $\mathcal{I}:=[0,1)$. Let $s \geq 1$ be a fixed dimension and denote by $\lambda_{s}$ the $s$-dimensional Lebesgue measure. For a set $U=\left\{\mathbf{u}_{0}, \ldots, \mathbf{u}_{N-1}\right\}$ of points in the $s$-dimensional unit cube $\mathcal{I}^{s}$ and for a Borel set $B \subset \mathcal{I}^{s}$ we define the local discrepancy by

$$
D(B, U):=\frac{1}{N} \sum_{0 \leq k<N} 1_{B}\left(\mathbf{u}_{k}\right)-\lambda_{s}(B),
$$

where $1_{B}$ denotes the indicator function of $B$. The discrepancy of $U$ is defined by $D(U):=\sup _{Q}|D(Q, U)|$, the supremum being taken over all subintervals $Q \subset \mathcal{I}^{s}$. The star discrepancy of $U$ is $D^{\star}(U):=\sup _{Q^{\star}}\left|D\left(Q^{\star}, U\right)\right|$, where $Q^{\star}$ runs through all subintervals of $\mathcal{I}^{s}$ of the form $\prod_{i=1}^{s}\left[0, a_{i}\right)$. A low-discrepancy point set in $\mathcal{I}^{s}$ is a set of $N$ points for which the discrepancy is of size $\mathcal{O}\left((\log N)^{s-1} / N\right)$, which is the minimum size possible. The most powerful current methods of constructing low-discrepancy point sets are based on the theory of $(t, m, s)$-nets. For an integer $b \geq 2$, an elementary interval in base $b$ is an interval of the form $\prod_{i=1}^{s}\left[a_{i} b^{-d_{i}},\left(a_{i}+1\right) b^{-d_{i}}\right)$, with integers $d_{i} \geq 0$ and $0 \leq a_{i}<b^{d_{i}}$ for $1 \leq i \leq s$. If $0 \leq t \leq m$ are integers, a $(t, m, s)$-net in base $b$ is a point set $U$ consisting of $b^{m}$ points in $\mathcal{I}^{s}$ such that $D(Q, U)=0$ for every elementary interval $Q$ in base $b$ with measure $b^{t-m}$. If $b \geq 2$ and $t \geq 0$ 
are integers, a sequence $\mathbf{u}_{0}, \mathbf{u}_{1}, \ldots$ of points in $\mathcal{I}^{s}$ is a $(t, s)$-sequence in base $b$ if, for all integers $j \geq 0$ and $m>t$, the points $\mathbf{u}_{\ell}$ with $j b^{m} \leq \ell<(j+1) b^{m}$ form a $(t, m, s)$-net in base $b$.

In the example of numerical integration, the QMC method achieves a significantly higher accuracy than the MC method, with the same computational effort. It may be hoped that the improvement obtained by using quasi-random points in place of random samples can also be attained in problems of numerical analysis that can be reduced to numerical integration. QMC simulations can outperform MC simulations in some applications: we refer to the IMACS Seminars on Monte Carlo Methods [1, 2, 4, 13].

In previous communications, we first proposed QMC schemes to simulate Markov chains with a discrete state space, either one-dimensional $[7,8]$ or multi-dimensional [3]. We next applied the method to one-dimensional continuous state spaces $[10,11]$. In the present work, we extend the QMC algorithm to Markov chains with continuous multi-dimensional state spaces.

\section{The method}

Our setting is an homogeneous Markov chain $\left\{X_{j}, j \in \mathbb{N}\right\}$ whose state space $E$ is a subspace of $\mathbb{R}^{s}$ for some $s \in \mathbb{N}^{*}$. The distribution $P_{0}$ of $X_{0}$ is known, and we assume that the chain evolves according to the stochastic recurrence:

$$
X_{j+1}=\varphi_{j+1}\left(X_{j}, U_{j+1}\right), \quad j \geq 0,
$$

where $\left\{U_{j}, j \geq 1\right\}$ is a sequence of i.i.d. uniform random variables over $\mathcal{I}^{d}$ for some $d \in \mathbb{N}^{*}$, and $\varphi_{j+1}: E \times \mathcal{I}^{d} \rightarrow E$ is a measurable map for each $j$.

To approximate the Markov chain by ordinary MC, we proceed as follows. Given a large integer $N$, we draw $N$ samples $\mathbf{x}_{k}^{0}, 0 \leq k<N$ from the initial distribution $P_{0}$. Then for each $k$, we generate a sample path of the chain via

$$
\mathbf{x}_{k}^{j+1}=\varphi_{j+1}\left(\mathbf{x}_{k}^{j}, \mathbf{u}_{k}^{j+1}\right), \quad j \geq 0
$$

where $\mathbf{u}_{k}^{1}, \mathbf{u}_{k}^{2}, \ldots$ are pseudo-random numbers which simulate independent and uniformly distributed random variables over $\mathcal{I}^{d}$. In order to construct a QMC algorithm for the approximation of the Markov chain, we reduce the simulation to numerical integration.

We denote by $\mathcal{M}^{+}$the set of all nonnegative measurable functions on $E$. If $P_{j}$ denotes the distribution of $X_{j}$, then

$$
\forall f \in \mathcal{M}^{+} \quad \int_{E} f d P_{j+1}=\int_{\mathcal{I}^{d}} \int_{E} f \circ \varphi_{j+1}(\mathbf{x}, \mathbf{u}) d P_{j}(\mathbf{x}) d \mathbf{u} .
$$


For $\mathbf{x} \in E$, let us write $\delta_{\mathbf{x}}$ for the unit mass at $\mathbf{x}$. We are looking for an approximation of $P_{j}$ of the form

$$
\widehat{P}_{j}:=\frac{1}{N} \sum_{0 \leq k<N} \delta_{\mathbf{x}_{k}^{j}},
$$

for some integer $N$ and a judiciously chosen set $X^{j}:=\left\{\mathbf{x}_{0}^{j}, \ldots, \mathbf{x}_{N-1}^{j}\right\} \subset E$. Let $b \geq 2, d_{1}, \ldots, d_{s}$ be integers and put $N:=b^{m}$ where $m=\sum_{i=1}^{s} d_{i}$. We shall use a low-discrepancy sequence $Y=\left\{\mathbf{y}_{0}, \mathbf{y}_{1}, \ldots\right\} \subset \mathcal{I}^{s+d}$ for QMC approximation. If $Y^{j}$ is the point set $\left\{\mathbf{y}_{\ell}: j N \leq \ell<(j+1) N\right\}$ and if $\pi^{\prime}$ and $\pi^{\prime \prime}$ are the projections defined by $\pi^{\prime}\left(u_{1}, \ldots, u_{s+d}\right):=\left(u_{1}, \ldots, u_{s}\right)$ and $\pi^{\prime \prime}\left(u_{1}, \ldots, u_{s+d}\right):=\left(u_{s+1}, \ldots, u_{s+d}\right)$, we assume that

$$
\forall j \in \mathbb{N} \quad \pi^{\prime} Y^{j} \text { is a }(0, m, s) \text {-net in base } b \quad \text { and } \quad \pi^{\prime \prime}\left(Y^{j}\right) \subset \stackrel{\circ}{\mathcal{I}}^{d},
$$

where $\stackrel{\circ}{\mathcal{I}}:=(0,1)$. For $\mathbf{u} \in \mathcal{I}^{s+d}$, we denote $\mathbf{u}^{\prime}:=\pi^{\prime}(\mathbf{u})$ and $\mathbf{u}^{\prime \prime}:=\pi^{\prime \prime}(\mathbf{u})$. We now explain our algorithm in which $N$ copies of the chain are simulated simultaneously.

\subsection{Generating the initial states}

A sample $X^{0}$ is chosen such that $\widehat{P}_{0} \approx P_{0}$. This means that $X^{0}$ has a small star $P_{0}$-discrepancy (see section 3 ).

\subsection{Transition}

Supposing that we have calculated a set $X^{j}$ of $N$ states such that $\widehat{P}_{j} \approx P_{j}$, we compute $X^{j+1}$ and $\widehat{P}_{j+1}$ in two steps.

\subsubsection{Relabeling the states}

The states are labeled $\mathbf{x}_{\mathbf{a}}^{j}$ using a multi-dimensional index in $\mathcal{A}:=\{\mathbf{a}=$ $\left.\left(a_{1}, \ldots, a_{s}\right), 0 \leq a_{i}<b^{d_{i}}, 1 \leq i \leq s\right\}$, such that:

if $a_{1}<a_{1}^{\prime}$ then $x_{\mathbf{a}, 1}^{j} \leq x_{\mathbf{a}^{\prime}, 1}^{j}$,

if $a_{1}=a_{1}^{\prime}, a_{2}<a_{2}^{\prime}$ then $x_{\mathbf{a}, 2}^{j} \leq x_{\mathbf{a}^{\prime}, 2}^{j}$,

if $a_{1}=a_{1}^{\prime}, \ldots, a_{s-1}=a_{s-1}^{\prime}, a_{s}<a_{s}^{\prime}$ then $x_{\mathbf{a}, s}^{j} \leq x_{\mathbf{a}^{\prime}, s}^{j}$. 


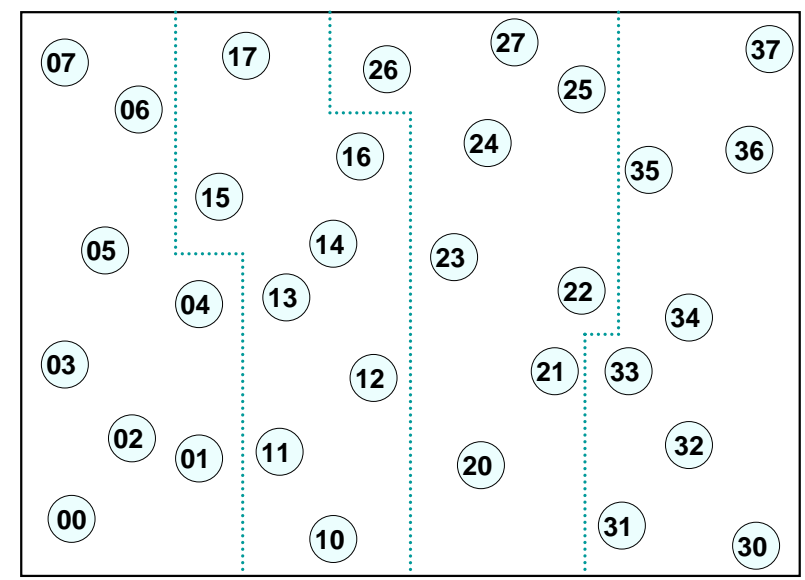

Figure 1: Relabeling the states $\left(b=2, s=2, d_{1}=2, d_{2}=3\right)$

Practically, we first sort the $N$ states in $b^{d_{1}}$ groups of size $N b^{-d_{1}}$ according to their first coordinates; then each group is sorted in $b^{d_{2}}$ subgroups of size $N b^{-d_{1}-d_{2}}$ by order of the second coordinates, and so on. The sorting is illustrated in Figure 1, with $b=2, s=2, d_{1}=2$ and $d_{2}=3$. Each circle represents a state and the numbers represent the pairs $\left(a_{1}, a_{2}\right)$. This type of sorting was first introduced in [6]. It provides a good description of the distribution of the states in the state space and will guarantee theoretical convergence: since each transition can be described by a numerical integration (see Section 2.2.2 below), the sorting reverts to minimizing the amplitude of the jumps of the function to be integrated.

\subsection{2. $Q M C$ integration}

If we replace $P_{j}$ by $\widehat{P}_{j}$ in the right-hand-side of (4), we define a probability measure $\widetilde{P}_{j+1}$ on $E$ :

$$
\int_{E} f d \widetilde{P}_{j+1}:=\int_{\mathcal{I}^{d}} \int_{E} f \circ \varphi_{j+1}(\mathbf{x}, \mathbf{u}) d \widehat{P}_{j}(\mathbf{x}) d \mathbf{u}, \quad f \in \mathcal{M}^{+} .
$$

This measure certainly approximates $P_{j+1}$, but it is not a sum of unit masses, like $\widehat{P}_{j}$. We recover this kind of approximation if we use a QMC quadrature rule. For $\mathbf{a}=\left(a_{1}, \ldots, a_{s}\right) \in \mathcal{A}$, let $\mathcal{I}_{\mathbf{a}}:=\prod_{i=1}^{s}\left[a_{i} b^{-d_{i}},\left(a_{i}+1\right) b^{-d_{i}}\right)$ and $1_{\mathbf{a}}$ be 
the indicator function of $\mathcal{I}_{\mathbf{a}}$. For $f \in \mathcal{M}^{+}$, define

$$
C^{j} f(\mathbf{u}):=\sum_{\mathbf{a} \in \mathcal{A}} 1_{\mathbf{a}}\left(\mathbf{u}^{\prime}\right) f \circ \varphi_{j+1}\left(\mathbf{x}_{\mathbf{a}}^{j}, \mathbf{u}^{\prime \prime}\right), \quad \mathbf{u}=\left(\mathbf{u}^{\prime}, \mathbf{u}^{\prime \prime}\right) \in \mathcal{I}^{s+d}
$$

Then we have

$$
\forall f \in \mathcal{M}^{+} \quad \int_{E} f d \widetilde{P}_{j+1}=\int_{\mathcal{I}^{s+d}} C^{j} f(\mathbf{u}) d \mathbf{u} .
$$

We retrieve $\widehat{P}_{j+1}$ if we perform a QMC approximation:

$$
\int_{E} f d \widehat{P}_{j+1}:=\frac{1}{N} \sum_{j N \leq \ell<(j+1) N} C^{j} f\left(\mathbf{y}_{\ell}\right), \quad f \in \mathcal{M}^{+} .
$$

The last step of the algorithm may be summarized as follows. For each $\mathbf{u}^{\prime} \in \mathcal{I}^{s}$, we associate the index $\mathbf{a}\left(\mathbf{u}^{\prime}\right):=\left(\left\lfloor b^{d_{1}} u_{1}\right\rfloor, \ldots,\left\lfloor b^{d_{s}} u_{s}\right\rfloor\right)$. From (6), the mapping $k \in\{j N, j N+1, \ldots,(j+1) N-1\} \rightarrow \mathbf{a}\left(\mathbf{y}_{k}^{\prime}\right) \in \mathcal{A}$ is one-to-one. The $N$ states $\mathbf{x}_{0}^{j+1}, \ldots, \mathbf{x}_{N-1}^{j+1}$ are computed according to:

$$
\mathbf{x}_{\mathbf{a}\left(\mathbf{y}_{\ell}^{\prime}\right)}^{j+1}=\varphi_{j+1}\left(\mathbf{x}_{\mathbf{a}\left(\mathbf{y}_{\ell}^{\prime}\right)}^{j}, \mathbf{y}_{\ell}^{\prime \prime}\right), \quad \text { for } j N \leq \ell<(j+1) N
$$

which must be compared with (3). This means that the projection $\pi^{\prime}\left(\mathbf{y}_{\ell}\right)$ of each point $\mathbf{y}_{\ell}$ of the low discrepancy sequence is used to select the state of the chain which will advance, while the remaining components $\pi^{\prime \prime}\left(\mathbf{y}_{\ell}\right)$ are used to determine the next state.

\section{Convergence}

First we adapt the basic concepts of QMC methods to the present study. If $U=\left\{\mathbf{u}_{0}, \ldots, \mathbf{u}_{N-1}\right\} \subset \mathcal{I}^{s}$ and if $c: \mathcal{I}^{s} \rightarrow \mathbb{R}$ is a non-negative measurable and bounded function, we put

$$
D(c, U):=\frac{1}{N} \sum_{0 \leq k<N} c\left(\mathbf{u}_{k}\right)-\int_{\mathcal{I}^{s}} c(\mathbf{u}) d \mathbf{u} .
$$

Let now $P$ be a probability measure on $E$ and $X:=\left\{\mathbf{x}_{0}, \ldots, \mathbf{x}_{N-1}\right\} \subset E$. For a measurable subset $A$ of $E$ we define the local $P$-discrepancy by

$$
D(A, X ; P):=\frac{1}{N} \sum_{0 \leq k<N} \chi_{A}\left(\mathbf{x}_{k}\right)-P(A),
$$


where $\chi_{A}$ denotes the characteristic function of $A$. The star P-discrepancy of the point set $X$ is defined by $D^{\star}(X ; P):=\sup _{\mathbf{z} \in E}\left|D\left(A_{\mathbf{z}}, X ; P\right)\right|$, where $A_{\mathbf{z}}:=\{\mathbf{x} \in E: \mathbf{x}<\mathbf{z}\}$ and $\mathbf{x}<\mathbf{z}$ means $\forall i x_{i}<z_{i}$. We shall also use the following notation: if $f \in \mathcal{M}^{+}$, then

$$
D(f, X ; P):=\frac{1}{N} \sum_{0 \leq k<N} f\left(\mathbf{x}_{k}\right)-\int_{E} f d P .
$$

The next Lemma is a version of the classical Koksma inequality [12].

Lemma 1. Let $P$ be a probability measure on $E$, with a Riemann-integrable density function $\rho$. Let $f: E \rightarrow \mathbb{R}$ be a function such that $f$ and $|f|$ are of bounded variation in the sense of Hardy and Krause. If $f$ or $\rho$ is continuous and if $X$ is a point set consisting of $N$ points in $E$, then

$$
|D(f, X ; P)| \leq V(f) D^{\star}(X ; P) .
$$

We now go back to the convergence analysis of the QMC algorithm. We restrict ourselves to the case $s=d$ and we assume that $E=\prod_{i=1}^{s} E_{i}$ and every $\varphi_{j}$ has the form: $\varphi_{j}\left(\mathbf{x}, \mathbf{u}^{\prime \prime}\right)=\left(\varphi_{j, 1}\left(x_{1}, u_{1}^{\prime \prime}\right), \ldots, \varphi_{j, s}\left(x_{s}, u_{s}^{\prime \prime}\right)\right)$. In addition, we assume that every $P_{j}$ has a continuous density function $\rho_{j}$.

Proposition 1. Suppose that

(i) $\forall j \geq 1 \forall \mathbf{z} \in E \forall \mathbf{u}^{\prime \prime} \in \mathcal{I}^{s} \quad V\left(\chi_{A_{z}} \circ \varphi_{j}\left(\cdot, \mathbf{u}^{\prime \prime}\right)\right) \leq 1$, and for every $j \geq 1$ and $1 \leq i \leq s$ :

(ii) for any $x_{i} \in E_{i}$, the $\operatorname{map} \varphi_{j, i}\left(x_{i}, \cdot\right): \stackrel{\circ}{\mathcal{I}} \rightarrow E_{i}$ is strictly increasing, (iii) for any $z_{i} \in E_{i}$, the $\operatorname{map} x_{i} \rightarrow\left(\varphi_{j, i}\left(x_{i}, \cdot\right)\right)^{-1}\left(z_{i}\right)$ is monotone.

Then

$$
\begin{aligned}
D^{\star}\left(X^{J} ; P_{J}\right) \leq & D^{\star}\left(X^{0} ; P_{0}\right)+b^{d_{1}+\cdots+d_{s-1}+\left\lfloor d_{s} / 2\right\rfloor} \sum_{j=0}^{J-1} D\left(Y^{j}\right) \\
& +\left(\frac{1}{b^{d_{1}}}+\cdots+\frac{1}{b^{d_{s-1}}}+\frac{1}{b^{\left\lfloor d_{s} / 2\right\rfloor}}\right) J
\end{aligned}
$$


Proof. For $j \geq 1, f \in \mathcal{M}^{+}$and $\mathbf{x} \in E$, denote $\Psi_{j} f(\mathbf{x}):=\int_{\mathcal{I}^{s}} f \circ$ $\varphi_{j}\left(\mathbf{x}, \mathbf{u}^{\prime \prime}\right) d \mathbf{u}^{\prime \prime}$. For $\mathbf{z} \in E$ we have:

$$
D\left(A_{\mathbf{z}}, X^{j+1} ; P_{j+1}\right)=D\left(\Psi_{j+1} \chi_{A_{\mathbf{z}}}, X^{j} ; P_{j}\right)+D\left(C^{j} \chi_{A_{\mathbf{z}}}, Y^{j}\right) .
$$

By Lemma 1 and assumption (i), we get $\left|D\left(\Psi_{j+1} \chi_{A_{\mathbf{z}}}, X^{j} ; P_{j}\right)\right| \leq D^{\star}\left(X^{j} ; P_{j}\right)$. The function $C^{j} \chi_{A_{\mathbf{z}}}$ is the indicator function of

$$
R_{\mathbf{z}}^{j}:=\bigcup_{\mathbf{a} \in \mathcal{A}} \mathcal{I}_{\mathbf{a}} \times\left\{\mathbf{u}^{\prime \prime} \in \mathcal{I}^{s}: \varphi_{j+1}\left(\mathbf{x}_{\mathbf{a}}^{j}, \mathbf{u}^{\prime \prime}\right)<\mathbf{z}\right\},
$$

hence $D\left(C^{j} \chi_{A_{\mathbf{z}}}, Y^{j}\right)=D\left(R_{\mathbf{z}}^{j}, Y^{j}\right)$. From (6) and (ii) we have $D\left(R_{\mathbf{z}}^{j}, Y^{j}\right)=$ $D\left(\widetilde{R}_{\mathbf{z}}^{j}, Y^{j}\right)$, where

$$
\widetilde{R}_{\mathbf{z}}^{j}:=\bigcup_{\mathbf{a} \in \mathcal{A}} \mathcal{I}_{\mathbf{a}} \times \prod_{i=1}^{s}\left[0,\left(\varphi_{j+1, i}\left(x_{\mathbf{a}, i}^{j}, \cdot\right)\right)^{-1}\left(z_{i}\right)\right) .
$$

Let $\delta_{s} \leq d_{s}$ be an integer. Denote for $\mathbf{z} \in E$ :

$$
\Phi_{\mathbf{a}}^{j+1}(\mathbf{z})=\left(\left(\varphi_{j+1,1}\left(x_{\mathbf{a}, 1}^{j}, \cdot\right)\right)^{-1}\left(z_{1}\right), \ldots,\left(\varphi_{j+1, s}\left(x_{\mathbf{a}, s}^{j}, \cdot\right)\right)^{-1}\left(z_{s}\right)\right) .
$$

Because the states are sorted and by (iii), there exist $s$ partitions of $[0,1]$ :

$$
\begin{aligned}
& 0=w_{0,1}^{j}(\mathbf{z}) \leq w_{1,1}^{j}(\mathbf{z}) \leq \cdots \leq w_{b^{d_{1}, 1}}^{j}(\mathbf{z})=1, \\
& \quad \cdots \\
& \quad=w_{\alpha_{1}, \ldots, \alpha_{s-1}, 0, s}^{j}(\mathbf{z}) \leq w_{\alpha_{1}, \ldots, \alpha_{s-1}, 1, s}^{j}(\mathbf{z}) \leq \cdots \leq w_{\alpha_{1}, \ldots, \alpha_{s-1}, b^{\delta_{s}, s}}^{j}(\mathbf{z})=1, \\
& \quad \text { for } 0 \leq \alpha_{1}<b^{d_{1}}, \ldots, 0 \leq \alpha_{s-1}<b^{d_{s-1}},
\end{aligned}
$$

such that, for $0 \leq \alpha_{1}<b^{d_{1}}, \ldots, 0 \leq \alpha_{s-1}<b^{d_{s-1}}, 0 \leq \alpha_{s}<b^{\delta_{s}}$ and $\alpha_{s} b^{d_{s}-\delta_{s}} \leq a_{s}<\left(\alpha_{s}+1\right) b^{d_{s}-\delta_{s}}$, we have

$$
\begin{aligned}
\Phi_{\alpha_{1}, \ldots, \alpha_{s-1}, a_{s}}^{j+1}(\mathbf{z}) \in \quad & {\left[w_{\alpha_{1}, 1}^{j}(\mathbf{z}), w_{\alpha_{1}+1,1}^{j}(\mathbf{z})\right] \times \cdots } \\
& \times\left[w_{\alpha_{1}, \ldots, \alpha_{s}, s}^{j}(\mathbf{z}), w_{\alpha_{1}, \ldots, \alpha_{s}+1, s}^{j}(\mathbf{z})\right] .
\end{aligned}
$$

If we put $\mathcal{J}_{\alpha}:=\prod_{i=1}^{s-1}\left[\alpha_{i} b^{-d_{i}},\left(\alpha_{i}+1\right) b^{-d_{i}}\right) \times\left[\alpha_{s} b^{-\delta_{s}},\left(\alpha_{s}+1\right) b^{-\delta_{s}}\right)$ and

$$
\begin{aligned}
\underline{Q}_{\mathbf{z}}^{j}:= & \bigcup_{\alpha} \mathcal{J}_{\alpha} \times\left[0, w_{\alpha_{1}, 1}^{j}(\mathbf{z})\right) \times \cdots \times\left[0, w_{\alpha_{1}, \ldots, \alpha_{s}, s}^{j}(\mathbf{z})\right), \\
\bar{Q}_{\mathbf{z}}^{j}:= & \bigcup_{\alpha} \mathcal{J}_{\alpha} \times\left[0, w_{\alpha_{1}+1,1}^{j}(\mathbf{z})\right) \times \cdots \times\left[0, w_{\alpha_{1}, \ldots, \alpha_{s}+1, s}^{j}(\mathbf{z})\right), \\
\partial Q_{\mathbf{z}}^{j}:= & \bigcup_{\alpha} \mathcal{J}_{\alpha} \times\left(\left[w_{\alpha_{1}, 1}^{j}(\mathbf{z}), w_{\alpha_{1}+1,1}^{j}(\mathbf{z})\right) \times \mathcal{I}^{s-1} \cup \cdots\right. \\
& \left.\cup\left[0, w_{\alpha_{1}, 1}^{j}(\mathbf{z})\right) \times \cdots \times\left[w_{\alpha_{1}, \ldots, \alpha_{s}, s}^{j}(\mathbf{z}), w_{\alpha_{1}, \ldots, \alpha_{s}+1, s}^{j}(\mathbf{z})\right)\right),
\end{aligned}
$$


then $D\left(\underline{Q}_{\mathbf{z}}^{j}, Y^{j}\right)-\lambda_{2 s}\left(\partial Q_{\mathbf{z}}^{j}\right) \leq D\left(\widetilde{R}_{\mathbf{z}}^{j}, Y^{j}\right) \leq D\left(\bar{Q}_{\mathbf{z}}^{j}, Y^{j}\right)+\lambda_{2 s}\left(\partial Q_{\mathbf{z}}^{j}\right)$. The subsets $\underline{Q}_{\mathbf{z}}^{j}$ and $\bar{Q}_{\mathbf{z}}^{j}$ are disjoint unions of $b^{d_{1}+\cdots+d_{s-1}+\delta_{s}}$ subintervals of $\mathcal{I}^{2 s}$, hence $\max \left(\left|D\left(\underline{Q}_{\mathbf{z}}^{j}, Y^{j}\right)\right|,\left|D\left(\bar{Q}_{\mathbf{z}}^{j}, Y^{j}\right)\right|\right) \leq b^{d_{1}+\cdots+d_{s-1}+\delta_{s}} D\left(Y^{j}\right)$. On the other hand, $\lambda_{2 s}\left(\partial Q_{\mathbf{z}}^{j}\right) \leq b^{-d_{1}}+\cdots+b^{-d_{s-1}}+b^{-\delta_{s}}$. By choosing $\delta_{s}=\left\lfloor d_{s} / 2\right\rfloor$, we get

$$
\left|D\left(\widetilde{R}_{\mathbf{z}}^{j}, Y^{j}\right)\right| \leq b^{d_{1}+\cdots+d_{s-1}+\left\lfloor d_{s} / 2\right\rfloor} D\left(Y^{j}\right)+\frac{1}{b^{d_{1}}}+\cdots+\frac{1}{b^{d_{s-1}}}+\frac{1}{b^{\left\lfloor d_{s} / 2\right\rfloor}} .
$$

The desired result follows by taking the supremum over $\mathbf{z} \in E$ and by induction on $j$.

\section{Numerical examples}

In this section, we present the results of numerical experiments which show the kind of improvement that our method can bring with respect to MC, even when the restrictive assumptions of Proposition 1 are not fulfilled. The examples we choose are artificial since the exact solutions are known and can be analytically calculated, but we use them as a benchmark to evaluate the viability of our method. The MC computations are done using the pseudorandom points generated by MRG32k3a of [9]. The QMC computations use Niederreiter's sequences in base $b=2[12]$.

\subsection{Asian option}

We consider the pricing of an Asian option on a single asset whose value $S(t)$ obeys: $d S(t)=r S(t) d t+\sigma S(t) d B(t)$, where $r$ is the risk-free interest rate, $\sigma$ the volatility parameter and $B$ is a standard Brownian motion (BM). Consider discrete observation times $0=t_{0}<t_{1}<\cdots<t_{J}=T$ and write:

$$
S\left(t_{j}\right)=S\left(t_{j-1}\right) \exp \left(\left(r-\sigma^{2} / 2\right) \delta t_{j}+\sigma \sqrt{\delta t_{j}} Z_{j}\right),
$$

where $\delta t_{j}:=t_{j}-t_{j-1}$ and $\left\{Z_{j}: j \geq 1\right\}$ is a sequence of i.i.d. standard normal variables. The value of the call option at maturity can be written as $C_{A}=$ $e^{-r T} \mathbb{E}\left[\max \left(\left(\prod_{j=1}^{J} S\left(t_{j}\right)\right)^{1 / J}-K, 0\right)\right]$ where the constant $K$ is the strike price. We want to estimate $C_{A}$ by our QMC algorithm and compare the results with those given by a classical MC scheme. Thus, we define a bi-dimensional Markov chain by: $X_{0}:=\left(S\left(t_{0}\right), 1\right)$ and $X_{j}:=\left(S\left(t_{j}\right),\left(\prod_{h=1}^{j} S\left(t_{h}\right)\right)^{1 / j}\right)$, for $j \geq 1$. Here $s=2, d=1$ and we consider the following parameters: $S(0)=$ $100, r=0.037, \sigma=0.2, T=240 / 365, K=90$. We estimate the error for 


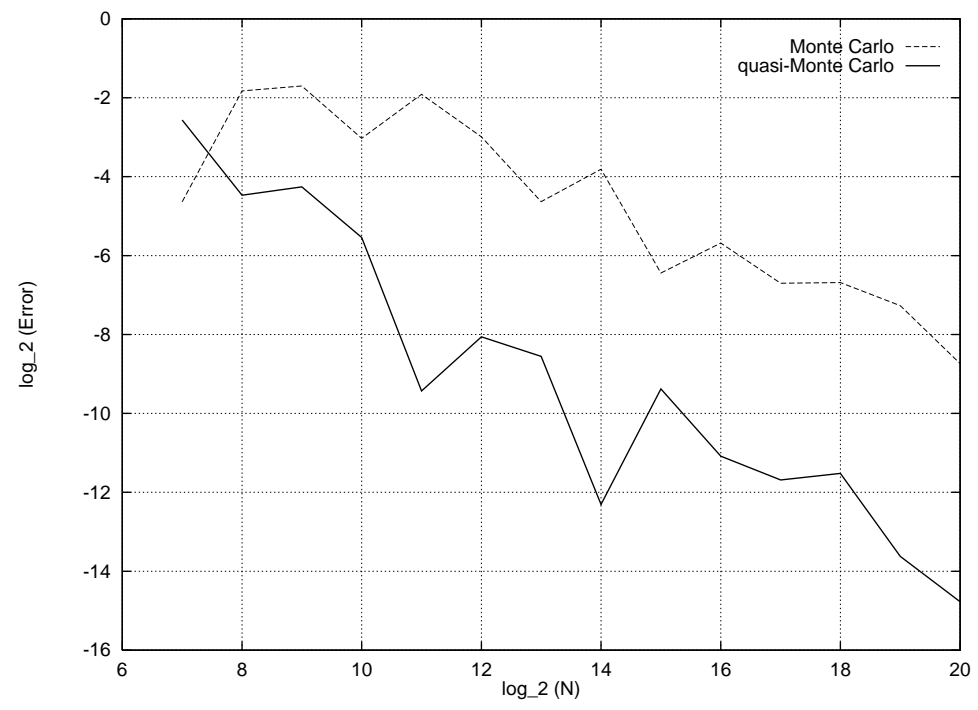

Figure 2: Asian option : the error as a function of $N$ for MC (thin line) and QMC (thick line)

$J=120$ as a function of $N$, say $\operatorname{Err}_{\mathrm{MC}}(N)$ for $\mathrm{MC}$ and $\operatorname{Err}_{\mathrm{QMC}}(N)$ for the QMC method. The value of $N$ varies from $2^{7}$ to $2^{20}$. Figure 2 shows the errors, in log-log scale. A linear regression analysis estimates the empirical convergence rate of the QMC method to be of the order of $\mathcal{O}\left(N^{-0.84}\right)$. Clearly, the QMC algorithm enjoys a much faster convergence than the MC scheme, whose convergence rate is known to be $\mathcal{O}\left(N^{-0.50}\right)$.

\subsection{European option on the maximum of two risky assets}

For our second example, we consider the pricing of an European call option on the maximum of two risky assets. The model is a bivariate geometric Brownian motion $S(t)=\left(S_{1}(t), S_{2}(t)\right)$ with interest rate $r$ and volatility parameters $\sigma_{1}$ and $\sigma_{2}$. Thus, for $i=1,2$ : $d S_{i}(t)=r S_{i}(t) d t+$ $\sigma_{i} S_{i}(t) d B_{i}(t)$, where $B_{1}$ and $B_{2}$ are two standard BM with correlation parameter $\rho$. For a strike price $K>0$, the option has discounted payoff $e^{-r T} \max \left(\max \left(S_{1}(T), S_{2}(T)\right)-K, 0\right)$ at maturity date $T>0$. The expected value $C_{M}$ of this payoff can be computed by formulas given in [5]. To estimate $C_{M}$, we discretize the problem using a set of observation times 


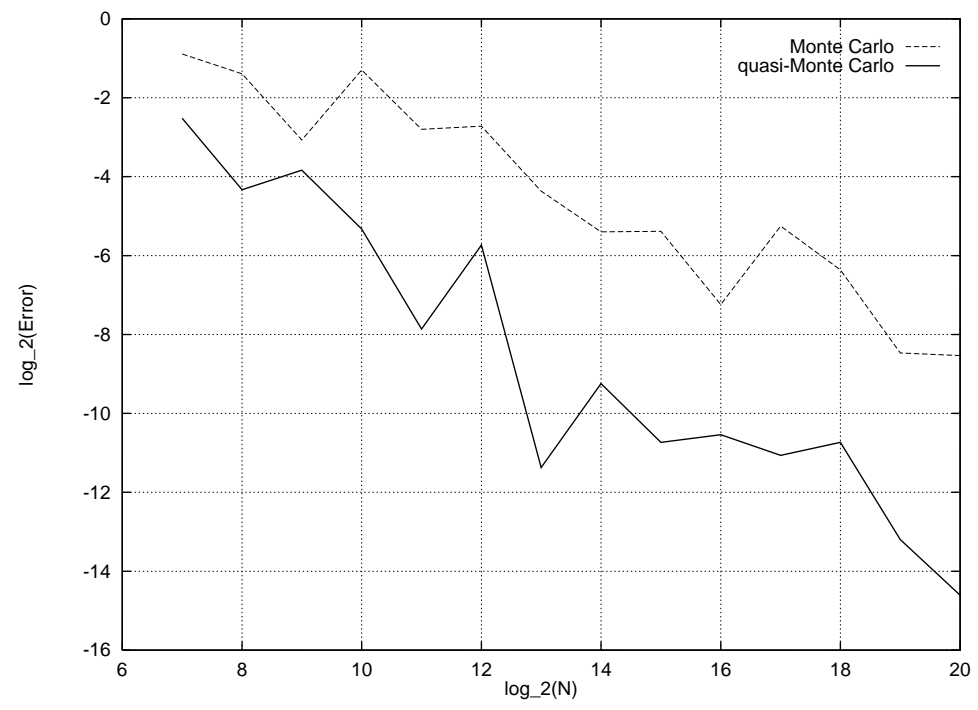

Figure 3: European option on the maximum of two risky assets : the error as a function of $N$ for $\mathrm{MC}$ (thin line) and QMC (thick line)

$0=t_{0}<t_{1}<\cdots<t_{J}$ and we simulate the assets as follows:

$$
\begin{aligned}
S_{1}\left(t_{j}\right)= & S_{1}\left(t_{j-1}\right) \exp \left(\left(r-\sigma_{1}^{2} / 2\right) \delta t_{j}+\sqrt{\delta t_{j}}\left(\sigma_{1} Z_{j, 1}\right)\right) \\
S_{2}\left(t_{j}\right)= & S_{2}\left(t_{j-1}\right) \\
& \cdot \exp \left(\left(r-\sigma_{2}^{2} / 2\right) \delta t_{j}+\sqrt{\delta t_{j}}\left(\sigma_{2} \rho Z_{j, 1}+\sigma_{2} \sqrt{1-\rho^{2}} Z_{j, 2}\right)\right),
\end{aligned}
$$

where $\left\{Z_{j}: j \geq 1\right\}$ are i.i.d. random variables such that $Z_{j} \sim \mathcal{N}\left(0, I_{2}\right)$ (here $I_{2}$ is the identity matrix). We define the Markov chain by $X_{j}=$ $\left(S_{1}\left(t_{j}\right), S_{2}\left(t_{j}\right)\right)$. Here $s=d=2$; for numerical illustration, let $S_{1}(0)=$ $S_{2}(0)=40, r=0.048, \sigma_{1}=0.2, \sigma_{2}=0.3, \rho=0.5, T=7 / 12, K=35$ and $J=100$. The number $N$ of paths varies from $2^{7}$ to $2^{20}$. The values of the errors are shown on Figure 3. Here again, regression analysis estimates the convergence speed to be $\operatorname{Err}_{\mathrm{QMC}}=\mathcal{O}\left(N^{-0.84}\right)$ for QMC, showing a strong improvement over MC. 


\section{Conclusion}

We have presented a QMC algorithm for the simulation of Markov chains with continuous and multi-dimensional state space. The method simulates several copies of the chain in parallel and reduces the error by sorting the states used in the simulation according to their successive coordinates at each step. Under certain assumptions, we have proved a convergence result as the number of simulated paths increases. The results of some numerical examples have shown that our QMC method is clearly superior to standard $\mathrm{MC}$ simulation in magnitude of error and in convergence rate. In the future, we shall analyze the convergence in more general settings and we shall provide some experiments with larger and more complicated models.

[1] R. Beauwens, A. Dubus, M. Hou (Eds.), Selection of papers presented at the IMACS Seminar on Monte Carlo Methods, Math. Comput. Simulation 47 (1998) 83-518.

[2] I. Dimov (Ed.), IMACS Sponsored Special Issue: The Second IMACS Seminar on Monte Carlo Methods, Math. Comput. Simulation 55 (2001) $1-305$.

[3] R. El Haddad, C. Lécot, P. L'Ecuyer, Quasi-Monte Carlo simulation of discrete-time Markov chains on multidimensional state spaces, in: S. Heinrich, A. Keller, and H. Niederreiter (Eds.), Monte Carlo and QuasiMonte Carlo Methods 2006, Springer-Verlag, Berlin, 2008, pp. 413-429.

[4] K. Entacher, W.C. Schmid, A. Uhl (Eds.), Special Issue: 3rd IMACS Seminar on Monte Carlo Methods, Math. Comput. Simulation 62 (2003) $217-571$.

[5] H. Johnson, Options on the maximum or the minimum of several assets, J. Financ. Quant. Anal. 22 (1987) 277-283.

[6] C. Lécot, A direct simulation Monte Carlo scheme and uniformly distributed sequences for solving the Boltzmann equation, Computing 41 (1989) 41-57.

[7] C. Lécot, B. Tuffin, Quasi-Monte Carlo methods for estimating transient measures of discrete time Markov chains, in: H. Niederreiter (Ed.), Monte Carlo and Quasi-Monte Carlo Methods 2002, Springer-Verlag, Berlin, 2004, pp. 329-343. 
[8] C. Lécot, B. Tuffin, Comparison of quasi-Monte Carlo-based methods for simulation of Markov chains, Monte Carlo Methods Appl. 10 (2004) $377-384$.

[9] P. L'Ecuyer, Good parameters and implementations for combined multiple recursive random number generators, Oper. Res. 47 (1999) 159-164.

[10] P. L'Ecuyer, C. Lécot, B. Tuffin, Randomized quasi-Monte Carlo simulation of Markov chains with an ordered state space, in: H. Niederreiter, D. Talay (Eds.), Monte Carlo and Quasi-Monte Carlo Methods 2004, Springer-Verlag, Berlin, 2006, pp. 331-342.

[11] P. L'Ecuyer, C. Lécot, B. Tuffin, A randomized quasi-Monte Carlo simulation method for Markov chains, Oper. Res. 56 (2008) 958-975.

[12] H. Niederreiter, Random Number Generation and Quasi-Monte Carlo Methods, SIAM, Philadelphia, 1992.

[13] K. K. Sabelfeld (Ed.), Selection of papers presented at the international conference: 4th IMACS Seminar on Monte Carlo Methods, Monte Carlo Methods Appl. 10 (2004) 183-655. 